\title{
Time-of-flight mass spectrometry to determine plasma temperature from ablated target surfaces
}

Eduardo Solís Meza, Lydia Rush, Carmen Menoni, Jorge Rocca, Greg Tallents, et al.

Eduardo Solís Meza, Lydia Rush, Carmen Menoni, Jorge Rocca, Greg Tallents, Erik Wagenaars, "Time-of-flight mass spectrometry to determine plasma temperature from ablated target surfaces," Proc. SPIE 11886, International Conference on X-Ray Lasers 2020, 1188610 (8 July 2021); doi: $10.1117 / 12.2593349$ 


\title{
Time-of-flight mass spectrometry to determine plasma temperature from ablated target surfaces
}

\author{
Eduardo Solís Meza*a, Lydia Rush ${ }^{b}$, Carmen Menoni $^{b}$, Jorge Rocca $^{a}$, Greg Tallents ${ }^{a}$, Erik \\ Wagenaars $^{a}$ \\ ${ }^{a}$ York Plasma Institute, Department of Physics, University of York, YO10 5DD, United \\ Kingdom; \\ ${ }^{b}$ Center for Extreme Ultraviolet Science and Technology and Department of Electrical and \\ Computer Engineering, Colorado State University, Fort Collins, Colorado 80523, U.S.A
}

\begin{abstract}
Extreme ultraviolet laser ablation mass spectrometry (EUV LA-MS), developed at Colorado State University, uses EUV laser wavelengths instead of traditional visible lasers, allowing sub-micron ablation spot sizes while maintaining a good sensitivity for trace element analysis. In this paper, we have explored the capabilities of this device as a non-perturbative technique to measure the temperature of the laser-produced plasma after the laser interaction. Mass spectra obtained from silver, aluminium, gold and silicon samples were used to identify and quantify the ion population created. Additionally, the ionization ratios were calculated and input in a coronal ionization equilibrium model to calculate the effective temperature of the plasma after the ablation process. Temperatures ranging 1.35 to $1.84 \mathrm{eV}$ were measured for the different materials, with heavier elements having lower temperatures than the lighter ones.
\end{abstract}

Keywords: Laser ablation,laser-produced plasma, mass spectrometry, EUV, TOF

\section{INTRODUCTION}

Laser ablation mass spectrometry (LA-MS) has been widely used as a reliable technique for the analysis of elemental composition of samples from diverse fields such as chemistry, biology and nuclear physics. This technique can be described as two independent tools that work together to analyse materials; a part of the system uses a laser to ablate a small part of the sample, inserting sample ions into the second part of the system, which analyses the masses of the sample ions. Traditionally, conventional lasers in the visible spectrum are used as ablation sources. In this regard, the laser source wavelength is proportional to the minimum ablation spot size achievable. In many cases, removing as little material as possible from the sample is critical, e.g. for biological or high-value samples.

Reducing the laser wavelength in laser sources for LA-MS is an evident solution to overcome this problem. However, the reduction in size and power consumption for laser sources at lower wavelengths have not evolved as fast as other components in mass spectrometers. Nevertheless, table-top sources in the extreme ultraviolet (EUV) range have been developed over the last decades providing a reliable and high repetition source of radiation in the nanometer range. The incorporation of this type of laser in a mass spectrometer, has recently been shown at Colorado State University. Specifically, this device uses a capillary discharge Ne-like Ar laser ${ }^{1}$ which produces pulses of $10 \mu \mathrm{J}$ and $1.5 \mathrm{~ns}$ duration at $46.9 \mathrm{~nm}$. It works together with a Time-of-flight (TOF) mass spectrometer. The reliability and high resolution of this device has been demonstrated in the analysis of well-characterized standard glasses using submicron ablation spots, where the results obtained were comparable with the analysis of the same material using a commercially available TOF secondary ion mass spectrometer $(\text { TOF SIMS })^{2}$. Furthermore, this new EUV TOF technique has shown its capabilities to create a 3D mapping of the chemical composition of different materials due to its unique capability to create submicron ablation spots ${ }^{3}$.

In this paper we explore the capabilities of the EUV TOF device to measure the temperature of the laserproduced plasma plume. The expanding plume is assumed to be in a coronal ionization equilibrium which allows the temperature to be estimated from the measured distribution of ions. This would provide a non-perturbative technique to measure plasma temperature.

*eduardo.solismeza@york.ac.uk

International Conference on X-Ray Lasers 2020, edited by Davide Bleiner,

Proc. of SPIE Vol. 11886, $1188610 \cdot$ (c) 2021 SPIE · CCC code:

$0277-786 \mathrm{X} / 21 / \$ 21 \cdot$ doi: $10.1117 / 12.2593349$

Proc. of SPIE Vol. 11886 1188610-1 


\section{EXPERIMENTAL METHOD}

The EUV TOF device used for the experiment was developed and built in the Engineering Research Center for Extreme Ultraviolet Science and Technology at the Colorado State University and it is composed of a compact EUV laser source and a TOF mass spectrometer array. Figure 1 shows a schematic view of the experimental arrangement including the laser source, optics to guide the pulse, the TOF tube and the detection zone. The compact source is based on a capillary discharge concept previously presented by Rocca et al. ${ }^{1}$ This source produces pulses of $46.9 \mathrm{~nm}, \sim 1.5 \mathrm{~ns}$ duration, $\sim 10 \mu \mathrm{J}$ energy and an annular beam profile. These pulses are directed onto the sample using a pair of toroidal mirrors and a zone plate array of $200 \mathrm{~nm}$ in the outer zone and a $50 \mu \mathrm{m}$ central opening. This zone plate converts the annular beam profile into Gaussian profiles on the sample. The central opening allows the extraction of ablated ions. The energy on the target surface is $\sim 10 \%$ of the EUV laser pulse energy, the spot size is $\sim 200 \mathrm{~nm}$ giving an intensity of $\sim 3 \times 10^{12} \mathrm{~W} / \mathrm{cm}^{2}$, which is just above the ablation threshold. The sample is positioned in the laser focus using a set of three axis motorized stages controlled independently. The samples are electrically biased using a $6 \mathrm{kV}$ potential grid to repel the positive ions created in the laser-produced plasma. After a grounded grid ,the ions travel different amounts of time inside the $\sim 1 \mathrm{~m}$ TOF tube depending on their different mass/charge ratio. At the end of the tube, a dual microchannel plate measures the ions in a time-resolved way.

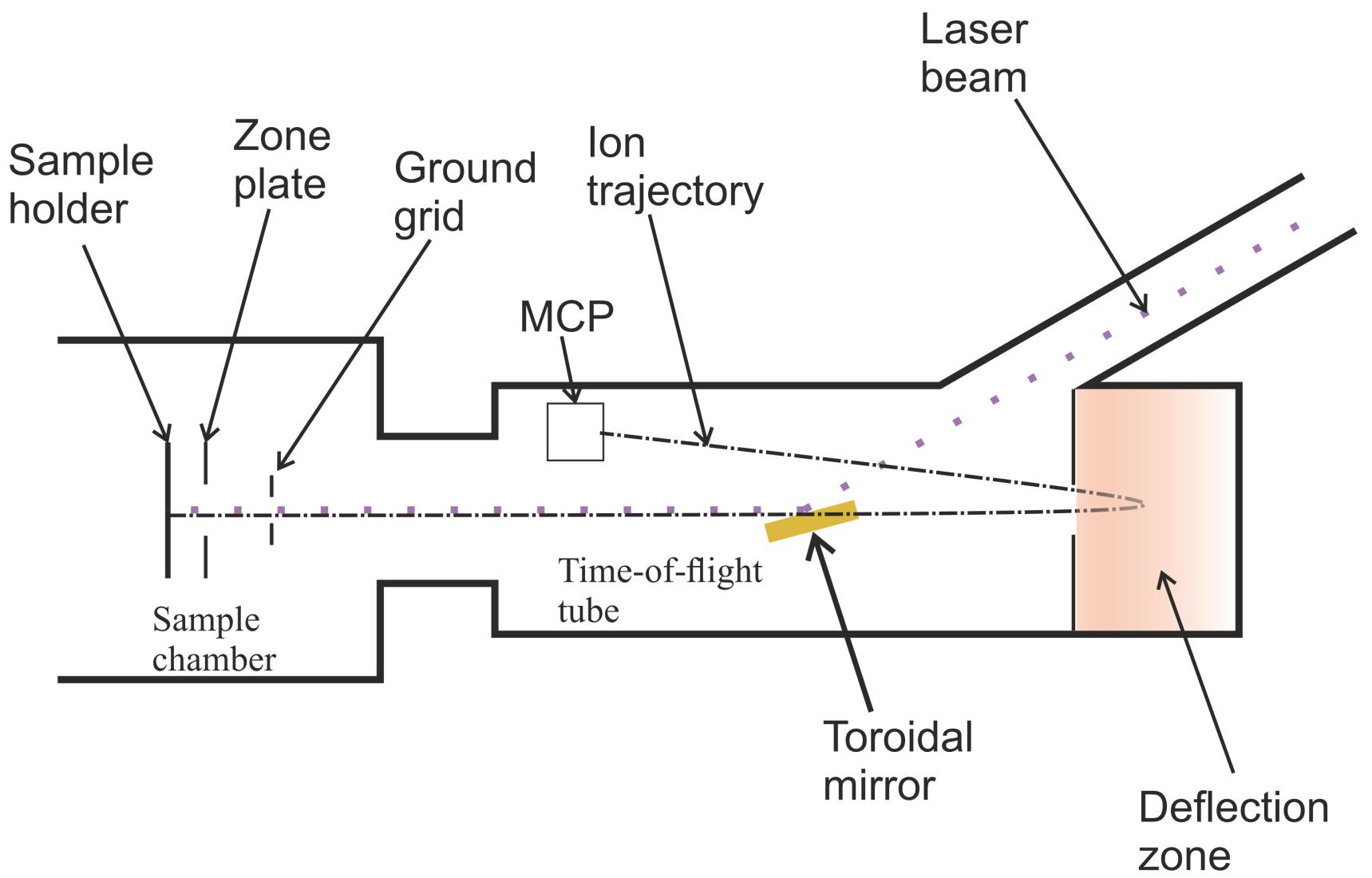

Figure 1: Schematic layout of the EUV TOF device. The purple dotted line represents the trajectory of the laser lasers, while the dashed line indicates the trajectory of the ions from the sample ${ }^{2}$.

The samples were shot in a matricial array of 10 by 10 ablation spots and each spot was ablated using 10 shots. The laser fluence was monitored and modulated using an in-line photoionization detector in an argon cell which also serves to attenuate the laser beam and change the laser fluence as required. This acquisition process is automatically controlled by software and the data acquired was analyzed using a custom-made python library. 
The materials ablated were gold, silver, aluminium and silicon. They were $2 \times 1 \mathrm{~mm}^{2}$ in size, $0.025 \mathrm{~mm}$ in thickness and mounted in a glass slide onto the sample holder.

\section{EXPERIMENTAL RESULTS}

The mass spectra obtained from the different materials analysed are presented in figures $2 \mathrm{a}-2 \mathrm{~d}$. These figures represent the average of 100 shots in the analysed samples. Figure 2a shows the mass spectrum for silver. The $1^{+}$state for two isotopes, ${ }^{107} \mathrm{Ag}$ and ${ }^{109} \mathrm{Ag}$, can be seen at 107 and $109 \mathrm{~m} / \mathrm{z}$, respectively. The peak located at 27 in figure $2 \mathrm{~b}$ corresponds to the first ionization state of ${ }^{27} \mathrm{Al}$. In figure $2 \mathrm{c}$, the peak at 197 identifies the $1^{+}$state for ${ }^{197} \mathrm{Au}$. Finally, in figure $2 \mathrm{~d}$ peaks corresponding to ${ }^{28} \mathrm{Si},{ }^{29} \mathrm{Si}$ and ${ }^{30} \mathrm{Si}$ can be identified. Higher ionization states can be identified for all the materials, as shown in the close-up plots for each material. Doubly ionized ions were found in all samples, while only for gold and silver $3^{+}$states were measured.

The remaining peaks at $\mathrm{m} / \mathrm{z}<50$ correspond to unavoidable contamination in the samples due to organic molecules deposition during handling and transportation. Aluminium and silicon samples form oxides in an open atmosphere as they are highly reactive with oxygen. ${ }^{18} \mathrm{O}$ molecules are observed in both samples as a consequence of oxygen detachment of these oxides induced by the laser. The $10-20 \mathrm{~m} / \mathrm{z}$ range peaks measured in all the samples correspond to the fragmentation patterns produced by organic compounds deposited in atmospheric conditions.

Even though the exact relation between the voltage measurements in the MCP plate and the absolute count of ions is not determined, it is most likely to be linearly proportional since the MCP response is constant at any mass. That would mean that the area under the peaks is directly proportional to the number of detected ions. For the determination of the temperature, ratios of densities of different ionisation stages are needed. In order to extract the relevant information from this data it is necessary to reduce the noise and extract the baseline of all these mass spectra. First the noise in the signal was identified and removed using a FFT noise reduction filter. Furthermore, a baseline correction method was implemented, specifically an asymmetric least square (ALS) smoothing process as proposed by Eilers ${ }^{4}$ and Oller-Moreno et al. ${ }^{5}$ These corrections helped to obtain an accurate numerical integration of the area under the peaks. 


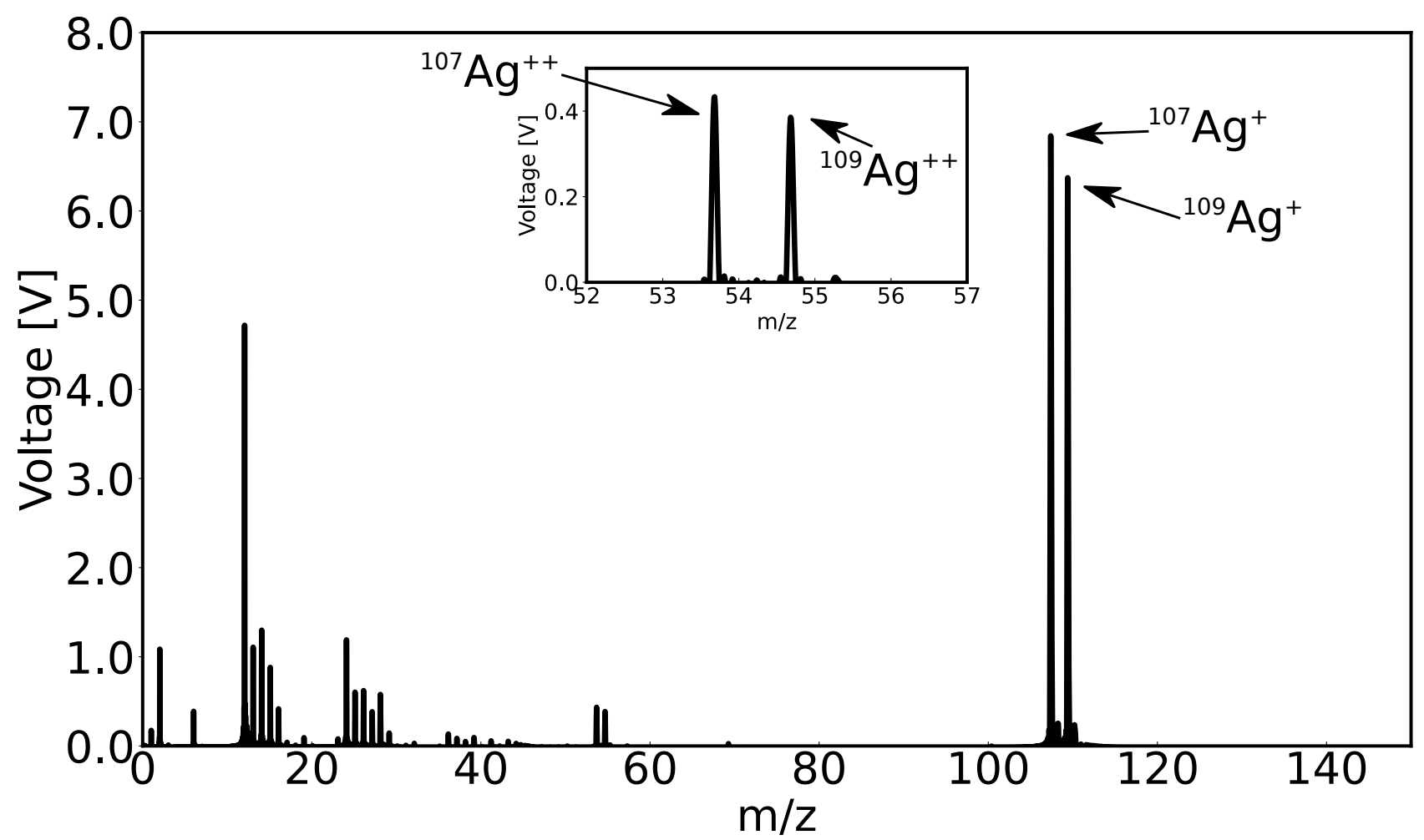

(a)

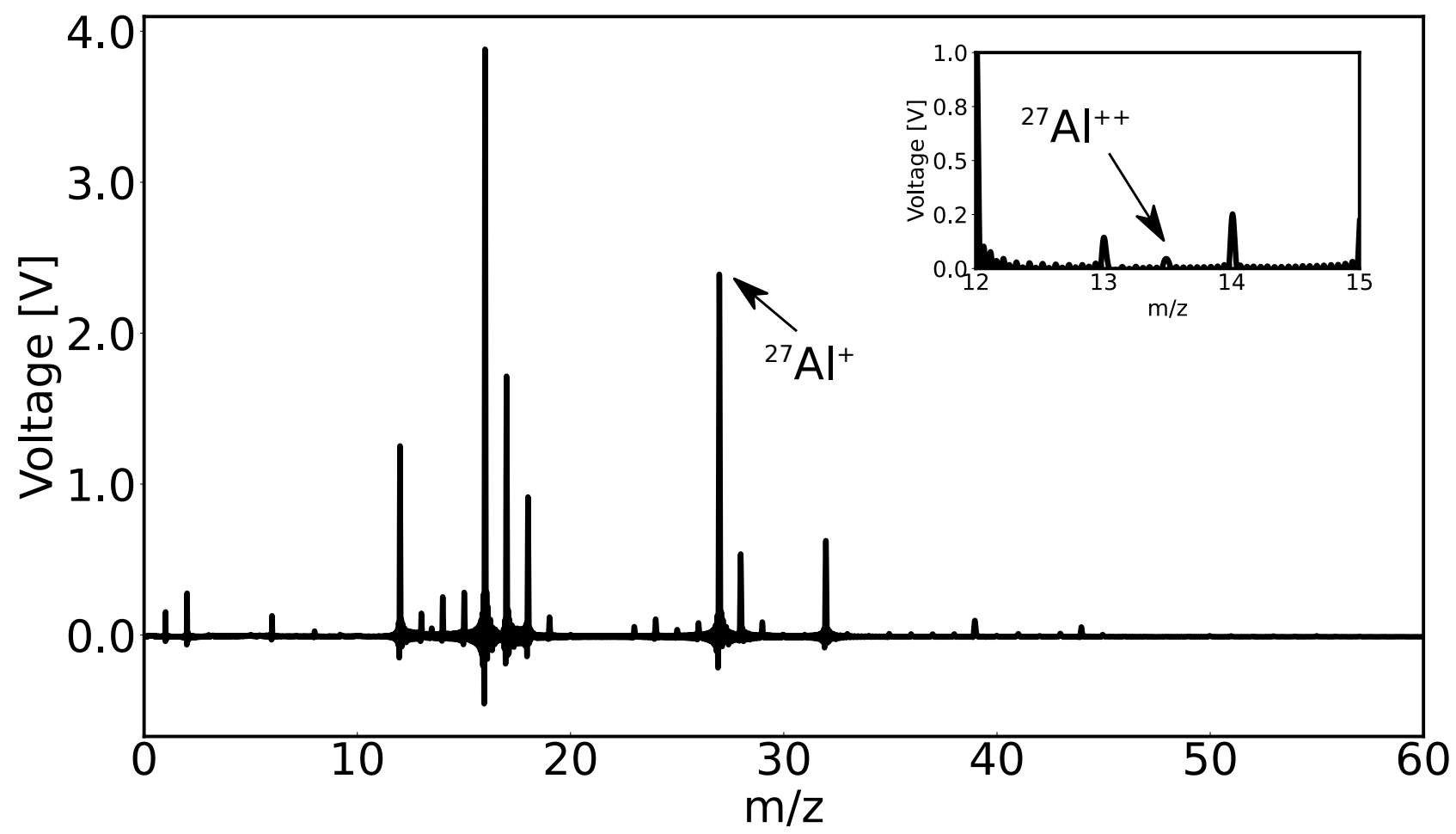

(b) 


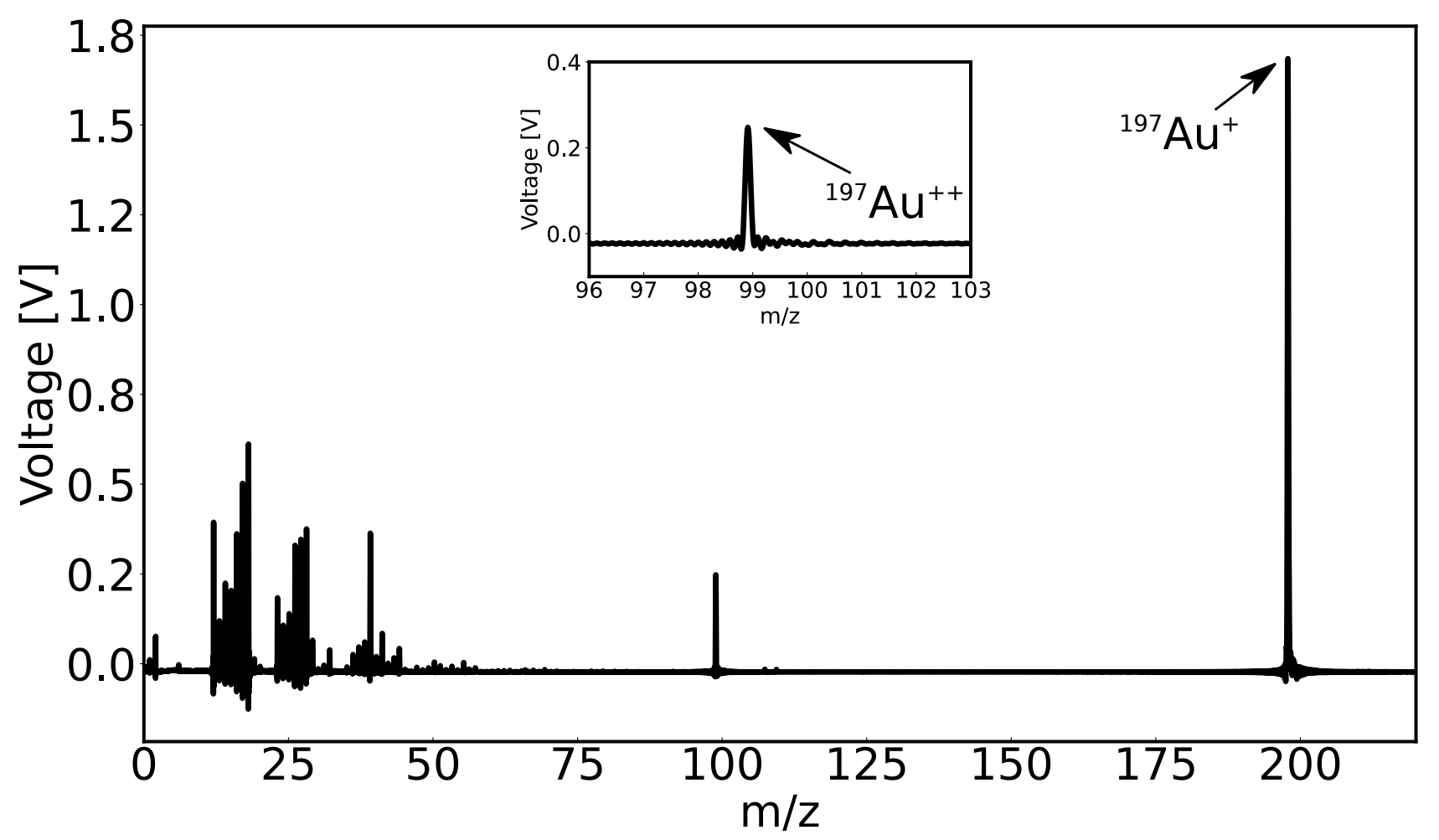

(c)

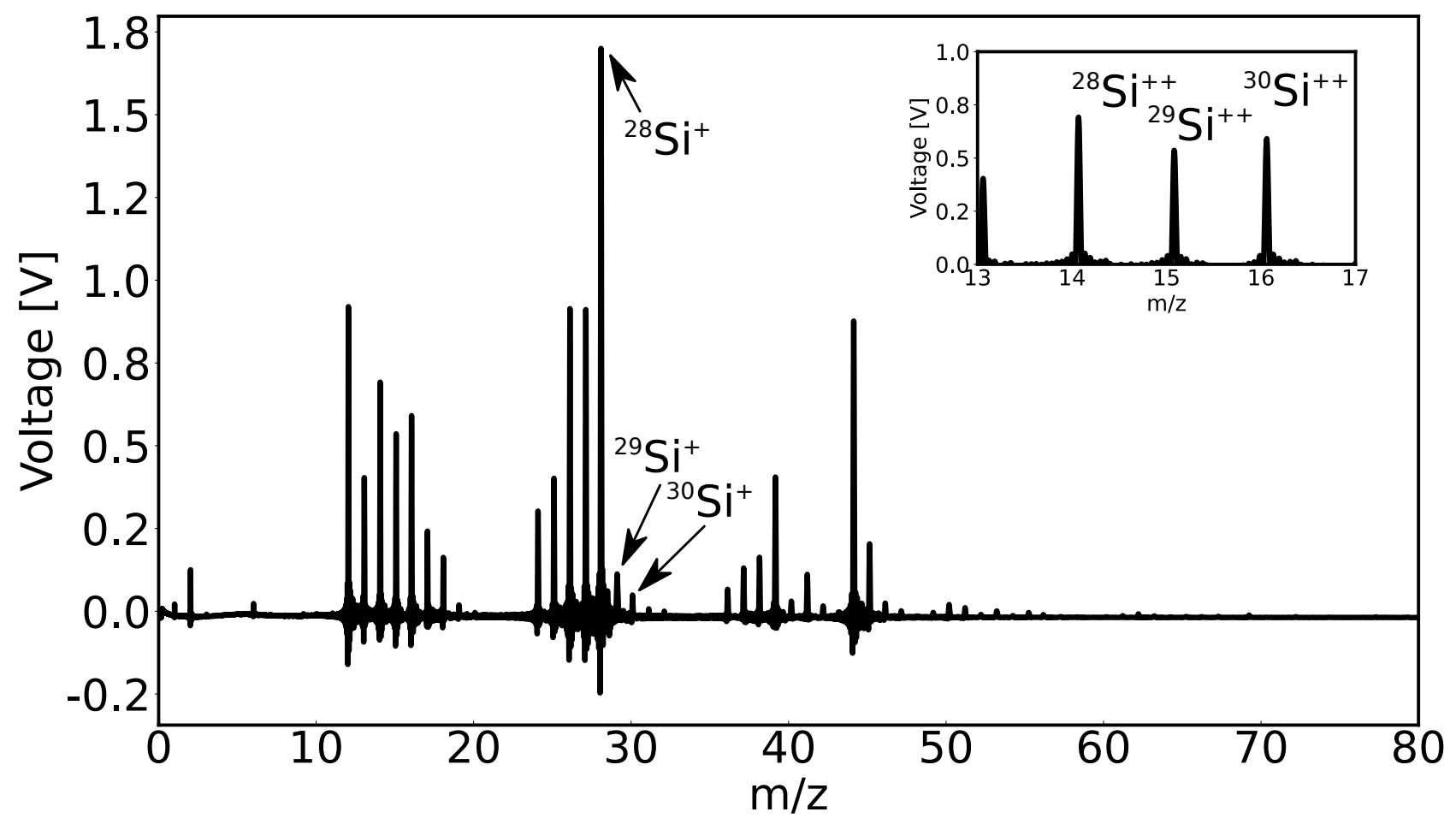

(d)

Figure 2: Mass spectra obtained for a) silver, b) aluminium, c) gold and d) silicon solid targets. These figures show the average mass spectra for multiple shots in the targets. 
The ratio of the areas under the peaks corresponding to $2^{+}$and $1^{+}$ionization states are presented in table 1. The plasma temperature was calculated using these ratios as an input for a coronal equilibrium model. This model is explained in the next section.

\begin{tabular}{|c|c|}
\hline Material & $n_{2} / n_{1}$ \\
\hline Silver & 0.05 \\
\hline Aluminium & 0.02 \\
\hline Gold & 0.24 \\
\hline Silicon & 0.20 \\
\hline
\end{tabular}

Table 1: Experimental ion ratio based on the integration of the different ion peaks measured in the EUV TOF.

\section{CORONAL MODEL}

The experimental conditions of the laser-plasma interaction; the low-intensity of the laser and the vacuum in the chamber, suggest that the plasma expansion is developed under adiabatic conditions and the plasma created should have a low electron density.

In the aforementioned case where the plasma density is low enough, the degree of ionization can be described as a coronal equilibrium state. In this equilibrium plasma state the radiative recombination and impact ionization processes are in dynamic balance. The degree of ionization depends exclusively on the electron temperature and is independent of the electron density ${ }^{6,7}$. For two ionization states, $\mathrm{i}$ and $\mathrm{i}+1$, this equilibrium can be described in terms of the collisional coefficient from ground state $\mathrm{K}_{1 C}$ and the radiative coefficient to ground state $\mathrm{A}_{C 1} \cdot{ }^{8}$

$$
n_{e} n_{i} K_{1 C}=n_{e} n_{i+1} A_{C 1}
$$

The ratio of ionization states is expressed as

$$
\frac{n_{i+1}}{n_{i}}=\frac{K_{1 C}}{A_{1 C}}
$$

Making the assumptions that the number of decay processes exceeds the number excitation processes for radiative transitions, and the number of particles in ground state is considerably greater than excited states, we can follow the procedure outlined by Tallents ${ }^{8}$ to establish expressions for the $K$ and $A$ coefficients and with this the ratio of ionization state densities.

The radiative recombination coefficient for free-bound recombination, $A_{C p}$, can be expressed as

$$
A_{C p}=\frac{4 \sqrt{2 \pi}}{\sqrt{3}}\left(\frac{e^{2}}{4 \pi \epsilon_{0}}\right)^{3} \frac{4}{3 c^{3}} \frac{1}{m_{0}^{\frac{3}{2}}} \frac{2 R_{d} Z_{i}^{4}}{\hbar n^{3}} \Theta(T)
$$

where $Z_{i}$ is the average ionization stage and $\Theta$ is a function of the temperature

$$
\Theta(T)=\tilde{G}_{f b}\left(\frac{1}{k_{B} T}\right)^{3 / 2} \exp \left(\frac{E_{i o n}}{k_{B} T}\right) E_{i}\left(\frac{E_{i o n}}{k_{B} T}\right)
$$

$\tilde{G}_{f b} \approx 1$ is the frequency averaged Gaunt factor for free-bound recombination and $E_{i}(y)$ is known as the exponential integral

$$
E_{i}(y)=\int_{y}^{\infty} \frac{e^{-x}}{x} d x
$$


The collisional ionisation coefficient from the ground state, $K_{p C}$, can be expressed as

$$
K_{p C}=2 \sqrt{\frac{2}{\pi m_{0}}}\left(\frac{1}{k_{B} T}\right)^{\frac{3}{2}} C_{i o n} g_{p}\left(\frac{k_{B} T}{E_{i o n}}\right) E_{i}\left(\frac{E_{i o n}}{k_{B} T}\right)
$$

where $C_{i o n}$ is a constant for collisional ionization with a value between $2.6-4.5 \times 10^{-18} \mathrm{~m}^{2}(\mathrm{eV})^{2}, g_{p}$ is the quantum-mechanical degeneracy weight.

Using the definitions for $A_{C 1}$ and $K_{1 C}$, equation 2 can be written as

$$
\frac{n_{i+1}}{n_{i}}=\frac{\sqrt{3}}{2 \pi} m_{0} C_{i o n} g_{p}\left(\frac{4 \pi \epsilon_{0}}{e^{2}}\right)^{3} \frac{3 c^{3}}{4} \frac{\hbar n^{3}}{2 R_{d} Z_{i}^{4}} \frac{1}{\tilde{G}_{f b}} \frac{k_{B} T}{E_{i o n}} \exp \left(-\frac{E_{i o n}}{k_{B} T}\right)
$$

The ratio of the ions experimentally measured in the TOF MS device show that the plasma is heavily dominated by $1^{+}$ions. In addition, because $Z_{i}$ is a slowly varying function of temperature, we assume $Z_{i}=1$. Subsequently, the experimental ion ratios were used in equation 7 to calculate the plasma temperature. This type of equation is known as a Lambert-W type function and it was solved using numerical methods. Table 2 shows the calculated plasma temperatures with this equation.

The accuracy of the temperature values can be estimated as $\sim 5 \%$ based on an error of up to $20 \%$ in the ionization ratio determined from the mass spectra.

\begin{tabular}{|c|c|c|}
\hline Material & $n_{2} / n_{1}$ & $\begin{array}{c}\text { Temperature } \\
(\mathrm{eV})\end{array}$ \\
\hline Silver & 0.05 & 1.84 \\
\hline Aluminium & 0.02 & 1.47 \\
\hline Gold & 0.24 & 1.72 \\
\hline Silicon & 0.20 & 1.35 \\
\hline
\end{tabular}

Table 2: Temperature calculation of the plasma.

\section{DISCUSSION AND CONCLUSIONS}

The temperatures presented in table 2 seem reasonable for the conditions of the laser. Moreover, there is consistency in the order of magnitude of the measured values across the materials, giving some confidence in the method. On closer inspection, higher temperatures are observed for the heavier materials Au and Ag. This is in line with what was calculated by Whittaker et al. ${ }^{9}$ for x-ray illumination of $\mathrm{C}$ and Fe.

These temperatures represent the plasma as it arrives at the entrance of the TOF tube, i.e. at the grounded grid, $3 \mathrm{~mm}$ from the sample surface. This means that significant plasma plume expansion has taken place by then.

It is even more important to note that the temperatures presented in table 2 are temperatures of an equivalent plasma in steady-state coronal equilibrium. In reality, there is a plasma with time-varying properties arriving at the grid position. Because the measurement is effectively time-integrated (on the time scales of the expansion), the measured ion ratio will represent an "effective temperature", not necessarily a true representation of the time-dependent plasma plume. In addition, the measured ions might be dominated by the most energetic ions created after the laser interaction, so-called fast ions. This could lead to an overestimate of the temperature.

Ongoing work is aimed at developing an adiabatic expansion model, similar to the one presented by Stapleton et al. ${ }^{10}$ to accurately reconstruct the (time-dependent) ion distributions arriving at the TOF grid. The modelling can be made to match the experimental ion ratios to learn more about the plasma behaviour in the ablation process. 


\section{REFERENCES}

[1] J.-J. Rocca, M. Frati, B. Benware, M. Seminario, J. Filevich, M. Marconi, K. Kanizay, A. Ozols, I. Artiukov, A. Vinogradov, and Y. Uspenskii, "Capillary discharge tabletop soft x-ray lasers reach new wavelengths and applications," Comptes Rendus de l'Académie des Sciences - Series IV - Physics 1(8), pp. 1065 - 1081, 2000.

[2] T. Green, I. Kuznetsov, D. Willingham, B. E. Naes, G. C. Eiden, Z. Zhu, W. Chao, J. J. Rocca, C. S. Menoni, and A. M. Duffin, "Characterization of extreme ultraviolet laser ablation mass spectrometry for actinide trace analysis and nanoscale isotopic imaging," Journal of Analytical Atomic Spectrometry, 2017.

[3] C. S. Menoni, I. Kuznetsov, T. Green, W. Chao, E. R. Bernstein, D. C. Crick, and J. J. Rocca, "Soft X-Ray Laser Ablation Mass Spectrometry for Chemical Composition Imaging in Three Dimensions (3D) at the Nanoscale," in Springer Proceedings in Physics, 2018.

[4] P. H. Eilers, "A perfect smoother," 2003.

[5] S. Oller-Moreno, A. Pardo, J. M. Jimenez-Soto, J. Samitier, and S. Marco, "Adaptive Asymmetric Least Squares baseline estimation for analytical instruments," in 2014 IEEE 11th International Multi-Conference on Systems, Signals and Devices, SSD 2014, 2014.

[6] P. G. Carolan and V. A. Piotrowicz, "The behaviour of impurities out of coronal equilibrium," Plasma Physics, 1983.

[7] R. J. Goldston, Introduction to plasma physics, CRC Press, 2020.

[8] G. Tallents, An introduction to the atomic and radiation physics of plasmas, Cambridge University Press, 2018.

[9] D. S. Whittaker, E. Wagenaars, and G. J. Tallents, "Temperatures following x-ray free-electron-laser heating of thin low- and medium- Z solid targets," Physics of Plasmas , 2011.

[10] M. W. Stapleton, A. P. McKiernan, and J. P. Mosnier, "Expansion dynamics and equilibrium conditions in a laser ablation plume of lithium: Modeling and experiment," Journal of Applied Physics, 2005. 